\title{
INFLUÊNCIA DE FATORES CLÍNICOS NA LESÃO RENAL AGUDA
}

\author{
INFLUENCE OF CLINICAL FACTORS ON ACUTE KIDNEY INJURY
}

\section{INFLUENCIA DE FACTORES CLÍNICOS EN LA LESIÓN RENAL AGUDA}

\author{
Tayse Tâmara Da Paixâo Duarte* \\ Luana Chagas Costa** \\ Wellington Luiz De Lima*** \\ Marcia Cristina Da Silva Magro****
}

\begin{abstract}
RESUMO
Objetivo: Verificar a relação entre variaçôes do clearance de creatinina $(\mathrm{ClCr})$ e os fatores clínicos de pacientes internados em clínica médica. Material e Método: Estudo longitudinal, do tipo coorte prospectivo, quantitativo, realizado em um hospital público do Distrito Federal. 85 pacientes evoluíram com Lesão Renal Aguda (LRA), de acordo com a classificação Kidney Disease: Improving Glogal Guidelines (KDIGO). O acompanhamento foi até 1 mês pós-alta hospitalar. A gravidade dos pacientes foi medida através do Índice de Comorbidade de Charlson. Considerou-se significativo resultados com $\mathrm{p} \leq 0,05$. Resultados: 51,8\% dos pacientes eram do sexo masculino. Entre as comorbidades se destacaram hipertensão arterial $(70,6 \%)$, diabetes mellitus $(57,6 \%)$ e as cardiopatias $(52,9 \%)$. O tempo de permanência hospitalar $(\mathrm{p}=0,001)$ e a idade $(\mathrm{p}=0,05)$ estiveram associadas a pior função renal. Pacientes com $\mathrm{ClCr} \leq 30 \mathrm{ml} / \mathrm{min}$ estiveram associados a uma maior taxa de mortalidade $(\mathrm{p}=0,007)$. Conclusão: Idade avançada e maior tempo de internação hospitalar se associaram a piora da função renal $(\mathrm{ClCr}<60 \mathrm{ml} / \mathrm{min})$. Àqueles com severa piora da função renal $(\mathrm{ClCr}<30 \mathrm{ml} / \mathrm{min})$ apresentaram maior taxa mortalidade.
\end{abstract}

Palavras-chave: Lesão renal aguda; Internação hospitalar; Fatores de risco.

\begin{abstract}
Objective: To verify the relationship between variations in creatinine clearance $(\mathrm{ClCr})$ and clinical factors in hospitalized patients in internal medicine. Material and Method: A prospective, quantitative, cohort study conducted at the medical clinic of a public hospital in Distrito Federal. 85 patients progressed with Acute Kidney Injury (AKI), according to the Kidney Disease Improving Glogal Guidelines (KDIGO) classification. Patients were followed-up to 1 month after hospital discharge. Patient severity was measured using the Charlson Comorbidity Index. Results with $\mathrm{p} \leq 0.05$ were considered significant. Results: $51.8 \%$ of the patients were male. Most common comorbidities were hypertension (70.6\%), diabetes mellitus $(57.6 \%)$ and heart disease

\footnotetext{
* Enfermeira, Doutoranda (c), Universidade de Brasília (UnB), Brasília, Brasil. Email: taysepaixao@unb.br. Autor Correspondente.

** Enfermeira, Faculdade de Ceilândia (FCE), Universidade de Brasília (UnB), Brasília, Brasil. Email: luchagas18@hotmail.com

***Enfermeiro, Mestrando (c), Universidade de Brasília (UnB), Brasília, Brasil. Email: wellingtonporteiras@hotmail.com

**** Enfermeira, Doutora, Faculdade de Ceilândia (FCE), Universidade de Brasília (UnB), Brasília, Brasil. Email: marciamagro@
} unb.br
\end{abstract}


(52.9\%). The length of hospital stay $(\mathrm{p}=0.001)$ and age $(\mathrm{p}=0.05)$ were associated with worse renal function. Patients with $\mathrm{CrCl} \leq 30 \mathrm{ml} / \mathrm{min}$ were associated with a higher mortality rate $(\mathrm{p}=0.007)$. Conclusion: Older patients and longer hospital stay were associated with worsening renal function $(\mathrm{CrCl}<60 \mathrm{ml} / \mathrm{min})$. Those with severe worsening of renal function $(\mathrm{CrCl}<30 \mathrm{ml} / \mathrm{min})$ presented higher mortality rate.

Keywords: Acute kidney injury; Hospitalization; Risk factors.

\section{RESUMEN}

Objetivo: Verificar la relación entre variaciones del clearance de creatinina (CLCr) y los factores clínicos de pacientes internados en medicina interna. Material y Método: Estudio longitudinal, del tipo cohorte prospectivo, cuantitativo, realizado en un hospital público del Distrito Federal. 85 pacientes evolucionaron con Lesión Renal Aguda (LRA), de acuerdo con la clasificación de las normas KDIGO (Kidney Disease: Improving Global Guidelines). El seguimiento fue hasta 1 mes post-alta hospitalaria. La gravedad de los pacientes fue medida a través del Índice de Comorbilidad de Charlson. Se consideraron significativos resultados con $\mathrm{p} \leq$ 0,05. Resultados: 51,8\% de los pacientes eran del sexo masculino. Entre las comorbilidades se destacaron hipertensión arterial $(70,6 \%)$, diabetes mellitus $(57,6 \%)$ y las cardiopatías $(52,9 \%)$. El tiempo de permanencia hospitalaria $(\mathrm{p}=0,001)$ y la edad $(\mathrm{p}=0,05)$ estuvieron asociadas a la peor función renal. Los pacientes con $\mathrm{ClCr}$ $\leq 30 \mathrm{ml} / \mathrm{min}$ estuvieron asociados a una mayor tasa de mortalidad ( $\mathrm{p}=0,007$ ). Conclusión: Edad avanzada y el mayor tiempo de internación hospitalaria se asociaron al empeoramiento de la función renal $(\mathrm{ClCr}<60 \mathrm{ml} /$ $\mathrm{min}$ ). Aquellos con severo empeoramiento de la función renal (CLCr $<30 \mathrm{ml} / \mathrm{min}$ ) presentaron mayor tasa de mortalidad.

Palabras clave: Lesión renal aguda; Internación hospitalaria; Factores de riesgo.

Fecha da recepção: 05/05/2019

\section{INTRODUÇÁO}

A Lesão Renal Aguda (LRA) é um problema de saúde pública de incidência crescente ${ }^{(1)}$ que acomete cerca de 7 a 18\% dos pacientes hospitalizados. Estimadamente, dois milhóes de pessoas ao ano, em todo mundo, morrem por comprometimento renal $^{(2)}$.

A LRA é de origem multifatorial, e quando combinada a comorbidades pré-existentes culmina em maior morbidade e mortalidade ${ }^{(3,4)}$. Estudos ressaltam que essa síndrome e seus efeitos clínicos adversos poderiam ser prevenidos ou atenuados por meio da detecção precoce e intervençóes capazes de limitar ou impedir sua progressão, resultando em melhor desfecho dos pacientes ${ }^{(5,6)}$.

A Taxa de Filtração Glomerular (TFG) ou clearence de creatinina $(\mathrm{ClCr})$ é considerado marcador sensível e específico de prejuízos na função renal ${ }^{(7)}$. A fórmula atual para cálculo do seu
Fecha da aceitação: 20/01/2020

valor estimado, recomendado pelo Kidney Disease Improving Glogal Guidelines (KDIGO), é obtida pela equação Chronic Kidney Disease Epidemiology Collaboration (CKD-EPI), onde a creatinina sérica ocupa lugar, haja vista ser ela o biomarcador de disfunção renal mais utilizado na prática clínica ${ }^{(8)}$.

Sabe-se que a TFG sofre interferência de alguns fatores como idade, sexo e raça. Além disso, a sua alteração pode resultar de complicaçóes decorrentes de doenças crônicas não transmissíveis (DCNT) como a hipertensão arterial sistêmica (HAS) e Diabetes Mellitus (DM) e ainda, maior tempo de internação. Evidências científicas reconhecem que a alteração da TFG está associada a piores desfechos e condiçóes clínicas de risco para desenvolvimento de LRA e Doença Renal Crônica (DRC) ${ }^{(7,9)}$.

O comprometimento progressivo ou persistente da funçáo renal representado pela doença renal em estágio terminal pode ocorrer após um episódio de $\operatorname{LRA}^{(10)}$. Sendo assim, a detecção precoce 
pela classificação $\mathrm{KDIGO}^{(8)}$ permite avaliação, e estratificação de risco fundamentada nas variaçóes da TFG ${ }^{(11)}$. Frente ao exposto, é inquestionável a importância da prevençẫo e detecção precoce da LRA no cenário hospitalar. Neste sentido, os profissionais de saúde, a exemplo dos enfermeiros, devem reconhecer os principais fatores de risco para LRA e implementar intervençóes específicas com intuito de reduzir a morbidade e a mortalidade associadas ao comprometimento renal, dessa forma melhorar a qualidade de vida dos pacientes e reduzir o impacto financeiro da LRA nos serviços de saúde ${ }^{(12)}$.

Neste sentido, o objetivo deste estudo foi verificar se há relação entre variações do clearance de creatinina e os fatores clínicos nos pacientes em regime de internação em clínica médica.

\section{MATERIAL E MÉTODO}

Estudo longitudinal, do tipo coorte prospectivo, quantitativo, realizado na unidade de clínica médica de um hospital público de grande porte do Distrito Federal. A instituiçấo onde se desenvolveu o estudo é referência na Regiáo Oeste do Brasil e atende a demanda populacional em diferentes especialidades clínicas.

Foram acompanhados 294 pacientes consecutivamente durante o período de 15 de janeiro de 2017 e 10 de março de 2018, segundo os critérios de elegibilidade. Como o estudo foi náo probabilístico consecutivo o pesquisador principal estabeleceu o período de coleta, e a partir disso foram incluídos todos os pacientes internados que atendessem os critérios de inclusão/exclusão, como amostra do estudo, durante o período fixado. No entanto, considerando as perdas por óbito ou desistência optou-se por prolongar o período de coleta por mais três meses.

Desse total, foram incluídos nesse estudo 85 que evoluíram com lesão renal, segundo a classificação $\mathrm{KDIGO}^{(8)}$. O acompanhamento do paciente perdurou até 1 mês após a alta hospitalar. Os dados foram obtidos por meio de um questionário estruturado com itens de identificaçáo (nome, idade, sexo, raça,) além de variáveis clínicas (doenças pregressas, doenças atuais) e laboratoriais (creatinina). Os pesquisadores responsáveis pela coleta de dados foram devidamente capacitados em relação ao preenchimento do questionário e a seleção dos participantes visando reduzir a possibilidade de viés de seleção e assegurar a validade interna do estudo.

Foram incluídos pacientes com idade $\geq 18$ anos, e ausência de história prévia de LRA, e excluídos aqueles com história de cirurgia de emergência, diagnóstico médico de doença renal crônica (DRC), em diálise, com história de transplante renal e aqueles em que a creatinina sérica não estava disponível.

O valor basal da creatinina sérica assumido para cada paciente foi o menor coletado nos primeiros 7 dias de internação na clínica médica ${ }^{(13)}$. Embora a classificação KDIGO seja constituída pelos critérios creatinina sérica e débito urinário, este último não foi utilizado, considerando que no setor onde se coletou os dados náo havia controle urinário diário dos pacientes em regime de internaçấo.

Os valores de referência adotados tanto para os exames laboratoriais quanto para as medidas hemodinâmicas seguiram o protocolo da Secretaria de Estado de Saúde do Distrito Federal (SES/DF) no momento em que os dados foram obtidos, a saber: creatinina sérica (masculino) 0,7-1,2 mg/dl; (feminino) $0,5-1,1 \mathrm{mg} / \mathrm{dl}^{2}$, pressão arterial sistólica (PAS) $120-80$ a $139-89 \mathrm{mmHg}$ e pressão arterial diastólica (PAD) $80-89 \mathrm{mmHg}^{\mathrm{b}}$.

$\mathrm{O}$ estágio de comprometimento renal foi identificado após cálculo do clearance de creatinina estimado por meio da equaçáo $\mathrm{CKDEPI}^{(7)}$ que utiliza como marcador principal a creatinina sérica para o cálculo. Sendo assim, dependendo da gravidade de acometimento renal o paciente foi classificado em estágio G1, aqueles com funçáo renal normal, estágio G2 função renal discretamente reduzida, ou ainda, estágio G3a, com comprometimento renal leve a moderadamente diminuído; estágio G3b aqueles com funçáo renal moderadamente a severamente diminuídas, estágio G4 severamente diminuído e grupo G5 com falência renal ${ }^{(14)}$.

\footnotetext{
a Valores obtidos no sistema interno (intranet) institucional da Secretaria de Saúde do Distrito Federal (DF), Brasil.

b Protocolo de Atenção à Saúde Manejo da Hipertensão Arterial Sistêmica e Diabetes Mellitus na Atenção Primária à Saúde. Portaria SES-DF No 161 de 21 de fevereiro de 2018, publicada no DODF No 37 de 23.02.2018. Disponível em: http:/www. saude.df.gov.br/wp-conteudo/uploads/2018/04/hipertencaoe-diabetes-Manejo_da_HAS_e_DM_na_APS.pdf
} 
Para avaliação da gravidade do quadro clínico do paciente, utilizou-se o Índice de Comorbidade de Charlson (ICC). Essa escala incorpora idade e 17 condiçóes médicas diferentes, sendo atribuído o valor (0 a 6) de acordo com as comorbidades de cada paciente. A soma dos pontos resulta no escore de comorbidade para o paciente ${ }^{(15)}$.

Para as variáveis quantitativas adotou-se o cálculo das medidas de centralidade (média, mediana) e de dispersão (desvio padrão e percentil 25-75). Os dados categóricos foram apresentados como frequência absoluta e relativa e analisados com o teste do qui-quadrado e o teste exato de Fisher, conforme apropriado. A normalidade foi testada com o teste de Kolmogorov-Smirnov. As distribuiçôes assimétricas foram descritas com medianas e intervalos interquartis e analisadas pelo teste Mann-Whitney. Foram também calculados o odds ratio (OR) e o respectivo intervalo de confiança de 95\% (IC 95\%) para a comparação de risco entre grupos. Com relação aos dados ausentes, realizaram-se análises de sensibilidade para avaliar diferenças nas características demográficas e clínicas, na taxa de lesão renal aguda e nos resultados secundários quando os dados sobrevivente versus não sobrevivente e do nível do clearance de creatinina estavam indisponíveis. Foi considerado significativo o resultado com $\mathrm{p} \leq 0,05(\alpha=5 \%)$.

$\mathrm{O}$ estudo foi submetido e aprovado pelo Comitê de Ética da Fundação de Ensino e Pesquisa em Ciências da Saúde (FEPECS) da Secretaria de Estado de Saúde do Distrito Federal sob o CAAE no 51576215.8.0000.5553. Atendendo aos aspectos éticos em pesquisa com seres humanos, com assinatura do Termo de Consentimento Livre e Esclarecido, com intuito de preservar a privacidade e confidencialidade dos participantes.

\section{RESULTADOS}

Dos 85 pacientes analisados a maioria foi do sexo masculino (44/51,8\%), com idade média de 66 \pm 14 anos e de raça/cor parda (56,5\%). Índice de Massa Corporal (IMC) $25,4 \mathrm{~kg} / \mathrm{m}^{2}, \mathrm{ClCr}=90 \mathrm{e}$ permanência média de hospitalização prolongada (49 dias). Em 72,9\% dos casos, os pacientes estavam conscientes e pouco mais da metade, acamados $(58,8 \%)$. Dentre as comorbidades apresentadas a hipertensão arterial sistêmica (HAS) foi identificada com maior frequência $(70,6 \%)$ (Tabela 1$)$.

Nos primeiros sete dias após identificação do comprometimento renal, a maioria dos pacientes foi classificado no estágio G3b (27,8\%), ou seja, com redução pouco severa da função renal. Entretanto, na alta hospitalar a maioria (26,3\%) apresentou estágio $\mathrm{G} 1$, demonstrando recuperação da função renal durante a hospitalização, situação que persistiu até um mês após a alta hospitalar $(44,4 \%)$ (Tabela 2).

Tabela 1. Distribuição dos pacientes de acordo com as características clínicas Distrito Federal, Brasil, 2018. $(\mathrm{n}=85)$.

\begin{tabular}{lcccc}
\hline Características & n (\%) & Média $( \pm$ DP) & Mediana $(\mathbf{2 5 - 7 5 \% )}$ \\
\hline IMC & & - & $25,4( \pm 6,7)$ & $24,8(21,2-27,7)$ \\
Clearance de Creatinina & - & $90( \pm 35)$ & $90(62-110)$ \\
Tempo de hospital (dias) & & - & $49( \pm 46)$ & $32(18-75)$ \\
\hline Mobilidade & Acamado & $50(58,8)$ & - & - \\
& Deambulante & $31(36,5)$ & - & - \\
\hline Comorbidades & Hipertensão & $60(70,6)$ & - & - \\
& Diabetes mellitus & $49(57,6)$ & - & - \\
& Cardiopatia & $45(52,9)$ & - & - \\
\hline
\end{tabular}


Tabela 2. Distribuição dos pacientes de acordo com a taxa de filtração glomerular (Clearance de creatinina), Distrito Federal, Brasil, 2018.

\begin{tabular}{llccc}
\hline Categoria & & $\begin{array}{c}\mathbf{7} \text { dias }(\mathbf{n}=\mathbf{7 9})^{\mathbf{1}} \\
\mathbf{n}(\mathbf{\%})\end{array}$ & $\begin{array}{c}\text { Alta }(\mathbf{n}=\mathbf{5 7})^{\mathbf{1}} \\
\mathbf{n}(\mathbf{\%})\end{array}$ & $\begin{array}{c}\mathbf{1} \text { mês pós-alta } \\
(\mathbf{n}=\mathbf{1 8})^{\mathbf{1}} \\
\mathbf{n}(\mathbf{\%})\end{array}$ \\
\hline G1 Função renal normal/ Alta & $(\mathrm{ClCr} \geq 90 \mathrm{ml} / \mathrm{min})$ & $10(12,7)$ & $15(26,3)$ & $8(44,4)$ \\
G2 Diminuição Ligeira & $(60 \leq \mathrm{ClCr}<90 \mathrm{~mL} / \mathrm{min})$ & $16(20,3)$ & $11(19,3)$ & $4(22,2)$ \\
G3a Diminuição Moderada & $(45 \leq \mathrm{ClCr}<60 \mathrm{~mL} / \mathrm{min})$ & $5(6,3)$ & $6(10,5)$ & $1(5,6)$ \\
G3b Diminuição pouco severa & $(30 \leq \mathrm{ClCr}<45 \mathrm{~mL} / \mathrm{min})$ & $22(27,8)$ & $10(17,5)$ & $1(5,6)$ \\
G4 Diminuição grave & $(15 \leq \mathrm{ClCr}<30 \mathrm{~mL} / \mathrm{min})$ & $20(25,3)$ & $12(21,1)$ & $4(22,2)$ \\
G5 Falência renal & $(\mathrm{ClCr} \leq 15 \mathrm{~mL} / \mathrm{min})$ & $6(7,6)$ & $3(5,3)$ & $0(0,0)$ \\
\hline
\end{tabular}

${ }^{1}$ Pacientes sobreviventes e com informação de exames laboratoriais.

Idade avançada $(\mathrm{p}=0,05)$ e tempo de internação prolongado $(\mathrm{p}=0,001)$ foram fatores que se associaram a pior função renal $(\mathrm{ClCr}<60 \mathrm{~mL} / \mathrm{min})$. Por outro lado, embora a maioria dos pacientes do sexo masculino apresente piora evolutiva da função renal não identificou-se correlação estatística ( $\mathrm{p}=$ $0,6)$. Esta situação se repetiu para a pressão sistólica e diastólica, raça, IMC, Índice de Comorbidade de Charlson e mobilidade (Tabela 3).

Pacientes que apresentaram função renal mais preservada, ou seja, com TFG $\geq 30 \mathrm{ml} / \mathrm{min}$ (Categoria G1 a G3a), evoluíram com maior taxa de sobrevida quando comparados àqueles de pior função renal TFG < 30 (Categorias G4 e G5) ( $p=$ 0,007 ) (Tabela 4). Isso foi representado pelo odds ratio de 4,19 (IC 95\%: 1,46 - 12,01), ou seja, a chance de evoluir a óbito dos pacientes da categorias G4 e G5 foi aproximadamente 4 vezes maior que dos demais pacientes.

\section{DISCUSSÃO}

Observou-se que o tempo de permanência hospitalar prolongado foi mais incidente em pacientes com idade avançada e pior função renal, ou seja, valores de clearance de creatinina menor que $60 \mathrm{ml} / \mathrm{min}$. Além disso, constatou-se que indivíduos com maior comprometimento renal $(\mathrm{ClCr}<30 \mathrm{ml} / \mathrm{min})$ possuem a chance de evoluir a óbito aproximadamente quatro vezes mais em relação aos demais pacientes.

O envelhecimento populacional e acúmulo de comorbidades interferem na taxa de filtração glomerular (TFG)/ Clearance de Creatinina (ClCr), o que tem predisposto nos últimos anos ao aumento da incidência de $\operatorname{LRA}^{(16,17)}$. O envelhecimento natural oferece modificações na estrutura e função renal, a exemplo da esclerose glomerular e fibrose intersticial, que podem culminar na lesão renal propriamente $\operatorname{dita}^{(11,18)}$ e tender à progressão para a cronicidade, doença renal em estágio final e morte $^{(19)}$, como identificado neste estudo. Esta situação se caracteriza pela redução progressiva da taxa de filtração glomerular ao longo da vida (aproximadamente $1 \mathrm{ml} / \mathrm{min} / 1,73 \mathrm{~m}^{2}$ por ano após os 30 anos de idade), e ainda de comorbidades e consequentes disfunçôes que comprometam a fisiologia renal ${ }^{(20)}$.

No presente estudo a idade avançada foi predominante, assim como a hipertensão arterial sistêmica (HAS), diabetes e cardiopatias, características clínicas que potencializam o risco para o comprometimento renal. Sabe-se que a HAS lesiona as unidades filtrantes dos rins, os néfrons, condição que limita ou impede a remoção de resíduos e excesso de líquido do sangue, predispondo à LRA $^{(6)}$. Enquanto a DM, desencadeia aumento progressivo da excreção urinária de albumina acompanhada do declínio da filtraçáo glomerular/ Clearance de creatinina ${ }^{(21)}$.

A identificação da maior gravidade clínica caracterizou-se pela elevação do Índice de Comorbidade de Charlson (ICC). Este perfil clínico favoreceu a maior incidência de LRA e pior desfecho, achado também observado em diferentes evidências científicas ${ }^{(22-24)}$. 


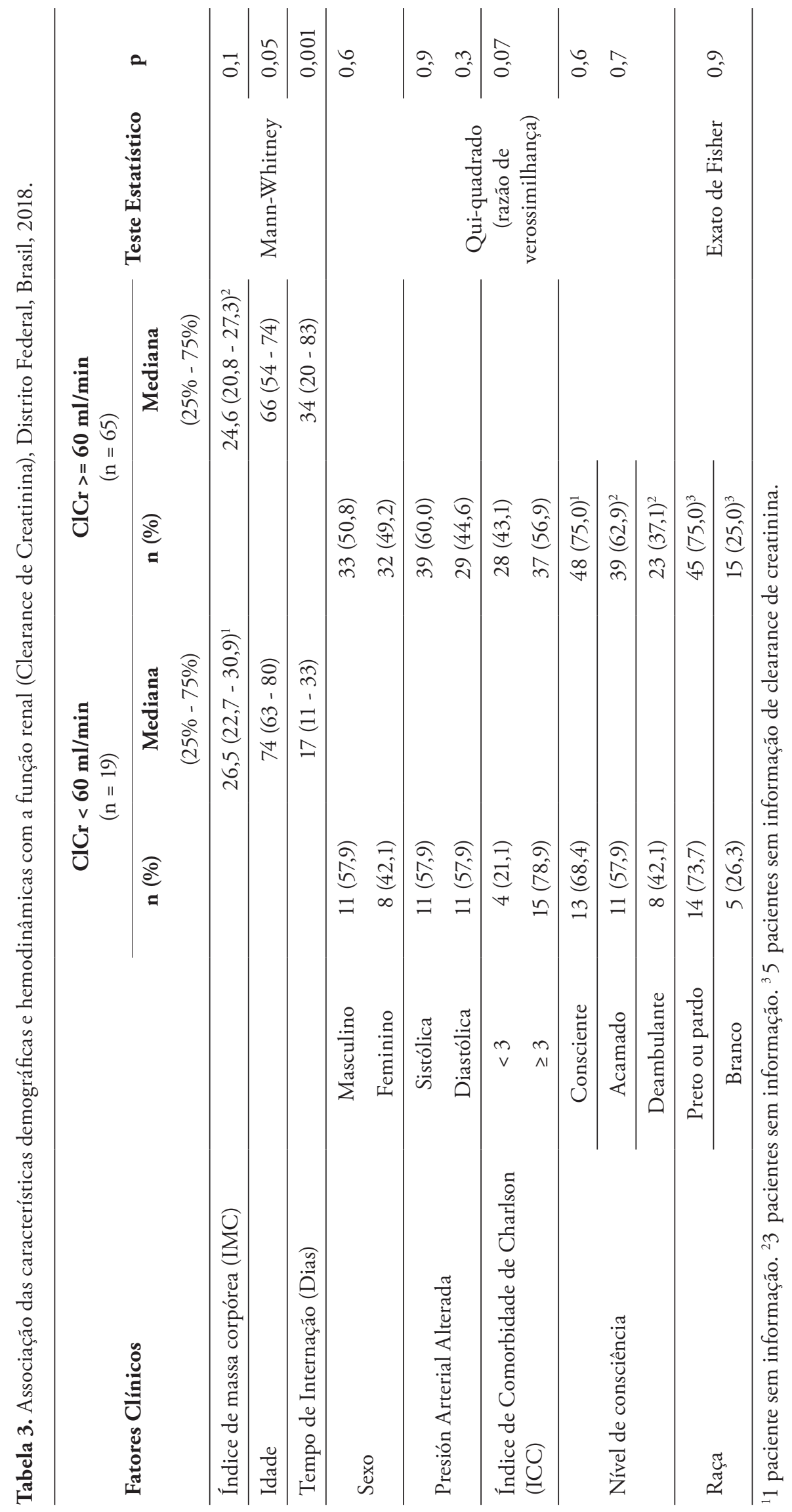


Tabela 4. Associação entre mortalidade de pacientes e a função renal (Clearance de Creatinina, ClCr), Distrito Federal, Brasil, 2018.

\begin{tabular}{lcccc}
\hline Classificação & $\begin{array}{c}\text { Sobrevivente }(\mathrm{n}=58)^{1} \\
\mathrm{n}(\%)\end{array}$ & $\begin{array}{c}\text { Não sobrevivente }(\mathrm{n}=21) \\
\mathrm{n}(\%)\end{array}$ & Teste Estatistico & $\mathrm{p}$ \\
\hline $\mathrm{G} 1$ a G4 $(\mathrm{ClCr} \geq 15 \mathrm{ml} / \mathrm{min})$ & $55(94,8)$ & $18(85,7)$ & \multirow{2}{*}{ Exato de Fisher } & 0,3 \\
$\mathrm{G} 5 \quad(\mathrm{ClCr} \leq 15 \mathrm{ml} / \mathrm{min})$ & $3(5,2)$ & $3(14,3)$ & & \\
\hline G1 a G3 $\quad(\mathrm{ClCr} \geq 30 \mathrm{ml} / \mathrm{min})$ & $44(75,9)$ & $9(42,9)$ & Qui-quadrado & 0,007 \\
G4 ou G5 $(\mathrm{ClCr}<30 \mathrm{ml} / \mathrm{min})$ & $14(24,1)$ & $12(57,1)$ & & \\
\hline
\end{tabular}

${ }^{1} 6$ pacientes sem informação de $\mathrm{ClCr}$.

A condição de acamado, identificada na maioria dos pacientes representa ao longo do tempo uma condição restritiva que predispóe a perda acentuada de massa magra pela inadequada oferta de substratos nutricionais, deflagrada por situações pró-inflamatórias, pró-oxidativas e de hipercatabolismo que potencializam a piora $\mathrm{da}$ função renal reconhecida pela redução do clearance de creatinina ${ }^{(25)}$. Condição clínica destacada por alguns estudos ${ }^{(26-28)}$ como disparadora do prolongamento de internaçóes e surgimento de complicaçóes que, por sua vez, podem interferir e retardar a recuperação da função renal ${ }^{(22)}$.

Essa permanência hospitalar prolongada pode agravar a LRA e acarretar piores desfechos clínicos $^{(4,}$ ), considerando que, além das comorbidades prévias, há por vezes necessidade de implementação de técnicas invasivas, terapêuticas medicamentosas, restrição da mobilidade dos indivíduos, o que resulta em maior risco de complicações e mortalidade ${ }^{(29)}$. Achado deste e de outros estudos ${ }^{(5,22,30)}$ reconhecido pela relação entre o maior tempo de internação, piora da função renal $(\mathrm{p}=0,001)$ e maior incidência de mortalidade $(\mathrm{p}=0,007)$.

Ademais, pacientes que sobrevivem com LRA têm risco aumentado de desenvolver DRC e doença renal terminal (DRT) a curto e a longo prazo $^{(2)}$, por isso o acompanhamento dos indivíduos com comprometimento renal é fundamental independentemente do grau aparente de recuperação ${ }^{(10)}$.

As limitaçóes deste estudo estão relacionadas à insuficiência de registros nos prontuários eletrônicos dos pacientes a falta de adesão dos pacientes participantes do estudo para avaliação da função renal após alta hospitalar. Por outro lado, a validade interna do estudo traduz a confiabilidade dos dados, assim como a possibilidade de generalização dos resultados.

Contudo, os dados obtidos podem subsidiar a equipe de enfermagem na construção e implementação de estratégias de intervenção individualizadas, pautadas na necessidade do paciente, cujo alvo é limitar ou impedir a progressão e a cronicidade da doença renal.

\section{CONCLUSÃO}

A idade avançada e o maior tempo de internação hospitalar foram fatores associados a piora $\mathrm{da}$ função renal, representada pela redução do clearance de creatinina $(\mathrm{ClCr}<60 \mathrm{ml} / \mathrm{min})$. Àqueles com severa piora da função renal $(\mathrm{ClCr}<30 \mathrm{ml} / \mathrm{min})$ apresentaram maior taxa de mortalidade.

\section{REFERÊNCIAS}

1. Cardoso BG, Carneiro TA, Magro MCS. Recuperação de pacientes com lesão renal aguda dialítica e não dialítica. Cogitare Enferm [Internet]. 2017 [citado 2019 fev 21]; 22(1): 1-9. Disponível em: http://www.saude.ufpr. $\mathrm{br} /$ portal/revistacogitare/wp-content/uploads/
sites/28/2017/01/48041-197534-1-PB.pdf

2. Chawla LS, Bellomo R, Bihorac A, Goldstein SL, Siew ED, Bagshaw SM, et al. Acute Disease Quality Initiative Workgroup 16. Acute kidney disease and renal recovery: consensus report of the Acute Disease Quality Initiative (ADQI) 16 Workgroup. Nat Rev Nephrol [Internet]. 2017 [citado $2019 \mathrm{fev}$ 21]; 13(4): 241-57. Disponível em: https://www. 
ncbi.nlm.nih.gov/pubmed/28239173

3. Zhang J, Tian J, Sun H, Digvijay K, Neri M, Bhargava V, et al. How Does Continuous Renal Replacement Therapy Affect Septic Acute Kidney Injury? Blood Purif [Internet]. 2018 [citado 2019 fev 23]; 46(4): 326-331. Disponível em: https:// www.karger.com/Article/FullText/492026

4. Joannidis M, Druml W, Forni LG, Groeneveld ABJ, Honore PM, Hoste E, et al. Prevention of acute kidney injury and protection of renal function in the intensive care unit: update 2017: Expert opinion of the Working Group on Prevention, AKI section, European Society of Intensive Care Medicine. Intensive Care Med [Internet]. 2017 [citado 2019 fev 23]; 43(6): 730-49. Disponível em: https://www.ncbi.nlm.nih.gov/pmc/articles/ PMC5487598/

5. Mehta RL, Burdmann EA, Cerdá J, Feehally J, Finkelstein F, García-García G, et al. Recognition and management of acute kidney injury in the International Society of Nephrology 0by25 Global Snapshot: a multinational cross-sectional study. Lancet [Internet]. 2016 [citado 2019 fev 23]; 387(10032): 2017-25. Disponível em: https:// www.ncbi.nlm.nih.gov/pubmed/27086173

6. Moura SLF, Duarte TTP, Magro MCS. Severity and outcome of patients with acute kidney injury in the intensive care unit. Rev enferm UFPE on line [Internet]. 2017 [citado 2019 mar 01]; 11(11): 4319-25. Disponível em: https://periodicos. ufpe.br/revistas/revistaenfermagem/article/ viewFile/23537/24677

7. Soares LO, Brune MFSS. Avaliação da função renal em adultos por meio da taxa de filtração glomerular e microalbuminúrica. Rev bras pesqui saúde [Internet]. 2017 [citado 2019 mar 01]; 19(3): 62-68. Disponível em: http://periodicos. ufes.br/RBPS/article/view/19566/13127

8. Kidney Disease: Improving Global Outcomes (KDIGO). Clinical Practice Guideline for Acute Kidney Injury. Kidney Int [Internet]. 2012 [citado 2019 mar 10]; 2 (Suppl 1): 1-138. Disponível em: https://kdigo.org/wp-content/uploads/2016/10/ KDIGO-2012-AKI-Guideline-English.pdf

9. Inker LA, Astor BC, Fox CH, Isakova $\mathrm{T}$, Lash JP, Peralta CA, et al. Commentary on the 2012 KDIGO clinical practice guideline for the evaluation and management of CKD. Am J Kidney Dis [Internet]. 2014 [citado 2019 mar 10]; 63(5): 713-35. Disponível em: https://www.ajkd.org/ article/S0272-6386(14)00491-0/pdf

10. Forni LG, Darmon M, Ostermann M, Oudemansvan Straaten HM, Pettilä V, Prowle JR, et al. Renal recovery after acute kidney injury. Intensive Care Med [Internet]. 2017 [citado 2019 mar 15]; 43(6):
855-66. Disponível em: https://www.ncbi.nlm. nih.gov/pmc/articles/PMC5487594/

11. Garasto S, Fusco S, Corica F, Rosignuolo $M$, Marino A, Montesanto A, et al. Estimating glomerular filtration rate in older people. Biomed Res Int [Internet]; 2014 [citado 2019 mar 15]; 2014: 916542. Disponível em: https://www.ncbi. nlm.nih.gov/pmc/articles/PMC3977451/

12. Hulse C, Davies A. Acute kidney injury: prevention and recognition. Nurs Times [Internet]. 2015 [citado 2019 mar 18]; 111(30-31): 12-5. Disponível em: https://www.ncbi.nlm.nih.gov/pubmed/26427253

13. Macedo E, Cerdá J, Hingorani S, Hou J, Bagga A, Burdmann E A, Rocco MV, Mehta RL. Recognition and management of acute kidney injury in children: The ISN 0by25 Global Snapshot study. PLoS One [Internet]. 2018 [citado 2019 mar 20]; 13(5): e0196586. Disponível em: https://www.ncbi.nlm. nih.gov/pmc/articles/PMC5929512/

14. Kidney Disease: Improving Global Outcomes (KDIGO). Clinical Practice Guideline for Lipid Management in Chronic Kidney Disease. Kidney Int [Internet]. 2013 [citado 2019 mar 20]; 3: 1-56. Disponível em: https://kdigo.org/wpcontent/uploads/2017/02/KDIGO-2013-LipidsGuideline-English.pdf

15. Charlson ME, Pompei P, Ales KL, Mackenzie CR. A new method of classifying prognostic comorbidity in longitudinal studies: development and validation. J Chron Dis [Internet]. 1987 [citado 2019 mar 20]; 40(5): 373-83. Disponível em: https://www.ncbi.nlm.nih.gov/pubmed/3558716

16. Rigonatto MCL, Magro MCS. Risk for acute kidney injury in primary health care. Rev Bras Enferm [Internet]. 2018 [citado 2019 mar 22]; 71(1): 205. Disponível em: http://www.scielo.br/pdf/reben/ v71n1/pt_0034-7167-reben-71-01-0020.pdf

17. Li Q, Zhao M, Wang X. AKI in the very elderly patients without preexisting chronic kidney disease: a comparison of 48-hour window and 7-day window for diagnosing AKI using the KDIGO criteria. Clin Interv Aging [Internet]. 2018 [citado 2019 mar 25]; 13:1151-60. Disponível em: https://www.ncbi.nlm.nih.gov/pmc/articles/ PMC6016022/

18. Pereira ER, Pereira AC, Andrade GB, Naghettini AV, Pinto FK, Batista SR, et al. Prevalence of chronic renal disease in adults attended by the family health strategy. J Bras Nefrol [Internet]. 2016 [citado 2019 mar 25]; 38(1): 22-30. Disponível em: http://www.scielo.br/pdf/jbn/ v38n1/en_0101-2800-jbn-38-01-0022.pdf

19. Humphreys BD, Cantaluppi V, Portilla D, Singbartl K, Yang L, Rosner MH, et al. Targeting Endogenous Repair Pathways after AKI. J Am Soc 
Nephrol [Internet]. 2016 [citado 2019 mar 28]; 27(4): 990-8. Disponível em: https://www.ncbi. nlm.nih.gov/pmc/articles/PMC4814191/

20. Almeida SLM, Silva KGN, Magro MCS. Risk of acute kidney injury in hypertensives and diabetics in the primary health care. Rev Enferm UFPE online [Internet]. 2016 [citado 2019 mar 28]; 10(9): 3197-202. Disponível em: https:// periodicos.ufpe.br/revistas/revistaenfermagem/ article/view/11398/13163

21. Domingueti CP, Fóscolo RB, Dusse LMS, Reis JS, Carvalho MDG, Gomes KB, et al. Association of different biomarkers of renal function with D-dimer levels in patients with type 1 diabetes mellitus (renal biomarkers and D-dimer in diabetes). Arch Endocrinol Metab [Internet]. 2018 [citado 2019 mar 30]; 62(1): 27-33. Disponível em: http://www.scielo.br/pdf/aem/v62n1/23593997-aem-62-01-0027.pdf

22. Ciriano ME, Porta JMP, Floristán CVV, García SO, Lipe RA, Floristán JMVV. Morbidity and mortality of acute renal failure in the Critical Care Unit of a regional hospital. Rev Esp Anestesiol Reanim [Internet]. 2018 [citado 2019 abr 03]; 65(6): 314-22. Disponível em: https://www.ncbi. nlm.nih.gov/pubmed/29598959

23. Wajner A, Zuchinali P, Olsen V, Carisi A, Polanczyk CA, Rodhe LE. Causes and Predictors of In-Hospital Mortality in Patients Admitted with or for Heart Failure at a Tertiary Hospital in Brazil. Arq Bras Cardiol [Internet]. 2017 [citado 2019 abr 03]; 109(4): 321-30. Disponível em: https://www. ncbi.nlm.nih.gov/pmc/articles/PMC5644212/

24. Hamzić-Mehmedbašić A, Rašić $S$, Balavac $M$, Rebić D, Delić-Šarac M, Durak-Nalbantić A. Prognostic indicators of adverse renal outcome and death in acute kidney injury hospital survivors. J Renal Inj Prev [Internet]. 2016 [citado 2019 abr 03]; 5(2): 61-8. Disponível em: https://www.ncbi.
nlm.nih.gov/pmc/articles/PMC4962671/pdf/jrip5-61.pdf

25. Berbel MN, Pinto MPR, Ponce D, Balbi AL. Aspectos nutricionais na lesão renal aguda Rev Assoc Med Bras [Internet]. 2011 [citado $2019 \mathrm{abr}$ 05]; 57(5): 600-606. Disponível em: http://www. scielo.br/pdf/ramb/v57n5/v57n5a22.pdf

26. Carrera-Lasfuentes P, Abad JM, Aguilar-Palacio I, Rabanaque MJ. Comorbidity as a predictor of health services utilization and mortality in patients with diabetes. Gac Sanit [Internet]. 2015 [citado 2019 abr 05]; 29(1): 10-4. Disponível em: https:// www.ncbi.nlm.nih.gov/pubmed/25200482

27. Pavkov ME, Harding LJ, Burrows NR. Trends in Hospitalizations for Acute Kidney Injury - United States, 2000 - 2014. MMWR Morb Mortal Wkly Rep [Internet]. 2018 [citado 2019 abr 05]; 67(10): 289-93. Disponível em: https://www.ncbi.nlm.nih. gov/pmc/articles/PMC5857198/pdf/mm6710a2. pdf

28. Benichel CR, Meneguin S. Fatores associados à lesão renal aguda em pacientes cirúrgicos na unidade de terapia intensiva. Rev Rene (Online) [Internet]. 2018 [citado 2019 abr 06]; 19: e3057. Disponível em: http://www.redalyc.org/ jatsRepo/3240/324054783001/324054783001. pdf

29. Coelho FUA, Watanabe M, Fonseca CD, Padilha KG, Vattimo MFF. Nursing Activities Score and Acute Kidney Injury. Rev Bras Enferm [Internet]. 2017 [citado 2019 abr 06]; 70(3): 475-80. Disponível em: http://www.scielo.br/pdf/reben/ v70n3/0034-7167-reben-70-03-0475.pdf

30. Szeto CC. Perspectives on acute kidney injury strategy: Hong Kong. Nephrology (Carlton) [Internet]. 2018 [citado 2019 abr 06]; 23 Suppl 4: 104-106. Disponível em: https://www.ncbi.nlm. nih.gov/pubmed/30298652 